\title{
A Method for Inoculation and Evaluation of Rice Sheath Blight Disease
}

\author{
Dong-Soo Park, Department of Plant Pathology, University of Arkansas, Fayetteville 72701, and National Yeong- \\ nam Agricultural Research Institute, Rural Development Administration, Neidong 1085, Milyang, Kyeongnam, Re- \\ public of Korea; Ronald J. Sayler, Department of Plant Pathology, University of Arkansas, Fayetteville; Yeon-Gyu \\ Hong and Min-Hee Nam, National Yeongnam Agricultural Research Institute, Rural Development Administration, \\ Republic of Korea; and Yinong Yang, Department of Plant Pathology, University of Arkansas, Fayetteville
}

\begin{abstract}
Park, D.-S., Sayler, R. J., Hong, Y.-G., Nam, M.-H., and Yang, Y. 2008. A method for inoculation and evaluation of rice sheath blight disease. Plant Dis. 92:25-29.

Sheath blight of rice, caused by Rhizoctonia solani, is one of the most important rice diseases worldwide; however, no rice cultivar has been found to be completely resistant to this fungus. To facilitate detailed analysis of sheath blight resistance at genetic, molecular, biochemical, and functional genomic levels, new methods were developed for effective and uniform infection and accurate evaluation of the disease. The efficiency of $R$. solani infection was tested on two resistant (Tetep and Jasmine 85) and two susceptible (Chucheongbyeo, Junambyeo) cultivars using three different inoculum types (agar block, liquid cultured mycelia ball, and mycelia suspension). By covering the inoculated sheaths with aluminum foil to maintain humidity, $100 \%$ infection rate was achieved in this study. Liquid cultured mycelia balls caused significantly longer lesions $(5.4 \mathrm{~cm})$ than other types of inoculum, including agar block $(2.4 \mathrm{~cm})$ and mycelia suspension $(1.6 \mathrm{~cm})$. An improved method for evaluating sheath blight disease was selected by comparing two methods for evaluating disease severity among three partially resistant cultivars and five susceptible cultivars inoculated with liquid cultured mycelia balls. In addition, a new formula was developed to calculate the disease susceptibility index. Lesion length and the susceptibility index generally were correlated in each leaf, but there were discrepancies between the two evaluation methods due to differences in plant architecture among the cultivars. The susceptibility index calculated using the new formula was the most accurate method for evaluating sheath blight disease across all cultivars. The effect of heading date and panicle number also was evaluated in relation to sheath blight resistance. Cultivars with late heading dates generally were more resistant to sheath blight than those with early heading dates.
\end{abstract}

Additional keywords: disease assay, host resistance

Sheath blight of rice, caused by the Rhizoctonia solani Kühn (teleomorph: Thanatephorus cucumeris (A.B. Frank) Donk), is a major disease of rice, second only to rice blast in reducing both grain yield and quality $(10,15,21)$. The pathogen is soilborne and has a wide host range, often infecting legume crops grown in rotation with rice $(10,16,21,28)$. Although no gene has been identified to confer complete resistance to sheath blight in rice, a

\section{Corresponding author: Y. Yang}

E-mail: yuy3@psu.edu

The first and second authors contributed equally to this work.

Current address of Y. Yang: Department of Plant Pathology, 405C Life Sciences Building, Pennsylvania State University, University Park 16802.

* The $e$-Xtra logo stands for "electronic extra" and indicates that a figure not included in the print edition is available online.

Accepted for publication 1 September 2007.

doi:10.1094/PDIS-92-1-0025

(C) 2008 The American Phytopathological Society number of genes have been reported to provide significant disease resistance (16). Resistance to sheath blight generally is considered to be polygenic $(11,18,24)$, with a wide variation in susceptibility levels among rice cultivars $(5,8,14)$. In the last two decades, several quantitative trait loci (QTL) have been detected based on co-segregation between DNA markers and disease resistance $(11,28)$; however, the validity of these QTL has been difficult because there was little agreement between the QTL identified by different groups (18). A number of environmental factors such as sunlight, humidity, temperature $(1,10,15,21)$, soil fertility (21), and silicon level in the soil (20) may contribute to the variation in the phenotype of disease resistance and make evaluation difficult. In addition, morphological traits such as plant height and heading date have been reported to affect the resistance of rice cultivars to sheath blight $(11,12,17,18,28)$.

Because vertical resistance is not available in rice against sheath blight, it is essential to have a technique that can differentiate incremental differences in quantitative, horizontal resistance among vari- ous cultivars, mutants, and transgenic lines. Many different inoculum sources have been used for studying rice sheath blight, including tooth picks colonized with $R$. solani $(19,20,28)$, colonized agar plugs $(1,8)$, infected rice grain-rice hull mixtures $(9,16,27)$, blended mycelia $(22,26)$, and sclerotia (25). Most of these inoculum sources introduce considerable variability into the infection process. Sclerotia, for example, vary widely with respect to their germination rate before infection based on their age and size (25). Each of these inoculum sources was used with its own inoculation method, either in the field or under controlled conditions.

In addition to a diversity of inoculation sources and methods used for the analysis of sheath blight resistance, different techniques for evaluating the severity of disease have been applied. For example, Groth et al. (4) first established the visual rating on a 0-to-9 scale with lesion length being estimated as a fraction of the total plant height. Eizenga and Lee (1) used two evaluation methods to quantify the severity of sheath blight. The first was a visual rating scale of 0 to 9 , modified from Savary and Mew (23), with the number corresponding to the percentage of leaves covered with lesions (13). The second evaluation technique these authors used was the median lesion length in the first one to three leaves from the base of the plant (1). The number of infection cushions and lesion type also have been used to differentiate the level of sheath blight resistance in rice lines $(3,9)$. Others have rated disease based on the ratio of the lesion area to the total leaf area (22). This study was undertaken to develop an effective, uniform, and reproducible technique for infecting rice plants with $R$. solani and to improve the method for accurately evaluating the disease severity. The morphological traits of heading date and panicle number also were analyzed in relation to sheath blight resistance.

\section{MATERIALS AND METHODS}

Plant materials. Rice cvs. Nipponbare, Jasmine 85, Chucheongbyeo, Junambyeo, Tetep, Taducan, IR50, Lemont, and 02428 were used in this study. Rice plants were grown in pots in a greenhouse $\left(28^{\circ} \mathrm{C}\right.$ day, $23^{\circ} \mathrm{C}$ night, $14 \mathrm{~h}$ of light) or under field conditions. Rice plants at late tillering 
stage (typically 10-week-old plants) were used for inoculation with $R$. solani.

Fungal inoculum. The fungal isolates (RR0140 and RS03-4) used in this study belong to the AG-1 IA group of $R$. solani. To compare the effects of inoculum types on the severity of sheath blight, three inoculum types were prepared and used, including (i) agar block (diameter $0.5 \mathrm{~cm}$ ), (ii) liquid cultured mycelia ball (diameter
$0.5 \mathrm{~cm}$ ), and (iii) 100- $\mu \mathrm{l}$ mycelial suspension $\left(10^{5} \mathrm{CFU} / \mathrm{ml}\right)$. After placing $R$. solani mycelia or sclerotia onto potato dextrose agar (PDA) and growth at room temperature $\left(22\right.$ to $\left.24^{\circ} \mathrm{C}\right)$ under continuous light (Fig. 1A), agar blocks (0.5-cm squares) were cut and prepared from the outer edge of a 3-day-old culture. Liquid cultures were started by inoculating $200 \mathrm{ml}$ of potato dextrose broth (PDB) medium in a
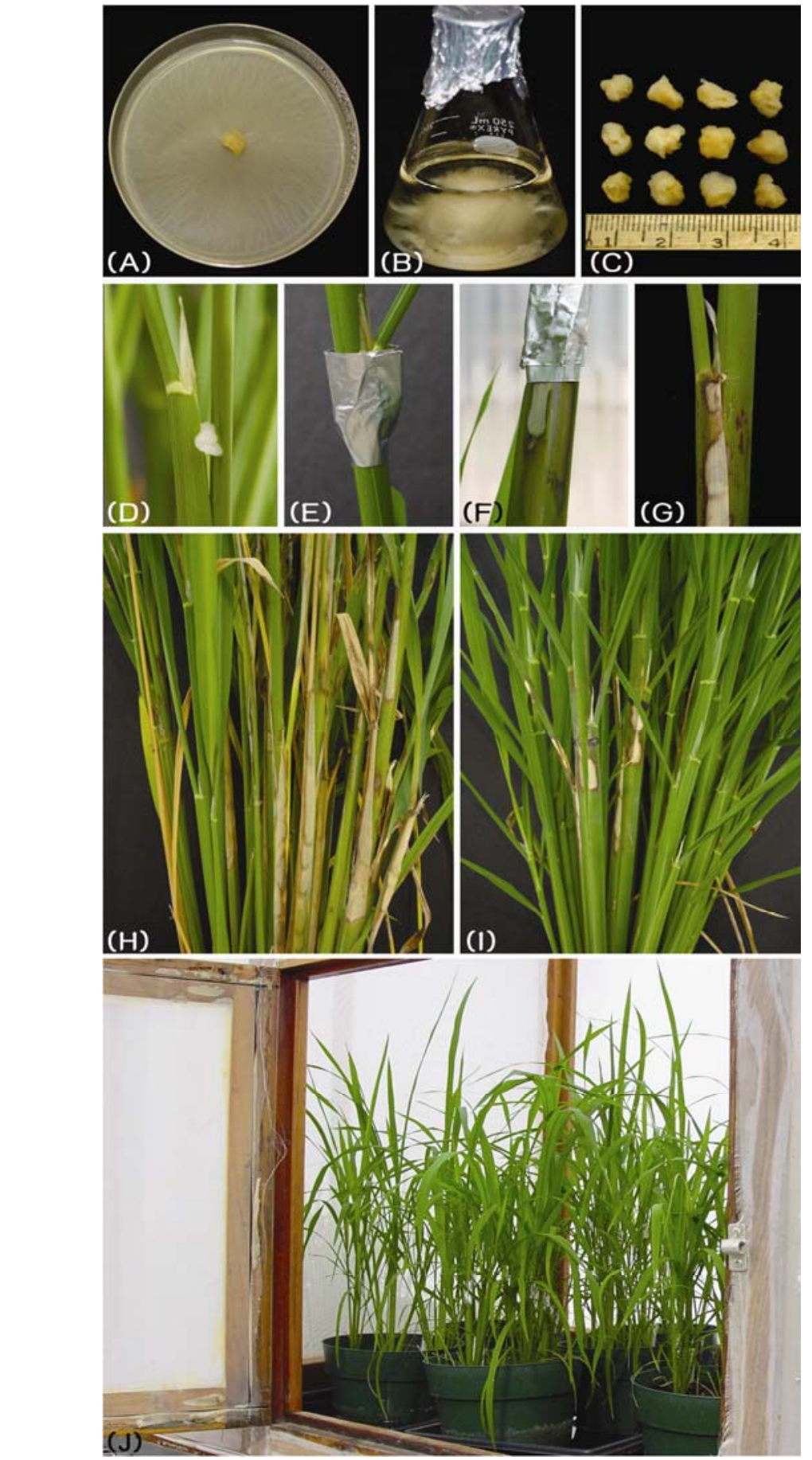

Fig. 1. Schematic representation of sheath blight inoculation: A, 3 days after inoculation of Rhizoctonia solani on potato dextrose agar; $\mathbf{B}, 7$ days after inoculation in potato dextrose broth; $\mathbf{C}$, mycelia balls harvested 7 days after inoculation; $\mathbf{D}$, inoculation of mycelia ball beneath leaf sheath; $\mathbf{E}$, inoculated sheath covered with aluminum foil; $\mathbf{F}$, appearance of disease symptoms 3 days after inoculation; $\mathbf{G}$, removal of aluminum foil; $\mathbf{H}$ and $\mathbf{I}, R$. solani-infected plants showing different level of infection between susceptible (Nipponbare) and resistant (Jasmine 85) cultivars, respectively; and $\mathbf{J}$, humidity chamber used to grow infected plants and maintain humidity between 80 and $100 \%$. 250-ml Erlenmeyer flask with a piece of fresh mycelia from PDA. After incubation on a shaker (130 to $140 \mathrm{rpm}$ ) under darkness for 7 to 10 days (Fig. 1B), liquid cultured mycelia were harvested and cut into small mycelial balls (approximately $0.5 \mathrm{~cm}$ in diameter; Fig. 1C) with forceps. The mycelial suspension was made by homogenizing liquid cultured mycelia with a blender.

Pathogen inoculation. Rice plants at late tillering stage were inoculated with $R$. solani by placing a mycelial ball beneath the leaf sheath (Fig. 1D). The inoculated sheath was covered immediately with aluminum foil (Fig. 1E). When typical lesions appeared after 3 days (Fig. 1F), the aluminum foil was removed (Fig. 1G). R. solaniinfected plants were left in a humidity chamber made of clear plastic for 3 weeks to allow for disease development (Fig. 1H and I). Plants were grown at $28^{\circ} \mathrm{C}$ under 14-h days in both the greenhouse and the humidity chamber. The humidity was maintained between 80 and $100 \%$ in humidity chambers from the time of inoculation to disease evaluation (Fig. 1J). Inoculations using the agar block (3-day cultures) and mycelial suspensions were the same as described for the mycelia ball. For the mycelial suspension, $100 \mu \mathrm{l}$ of homogenized mycelium was applied for each inoculation using a pipette. Seven days after inoculation, the lesion length on the sheath of the inoculated plants was measured. Ten replications (culms) were used for each inoculum type in the inoculum study. The results were verified by repeating the experiment two times.

Disease evaluation. To evaluate sheath blight resistance or susceptibility of rice cultivars, the lesion length and the degree of disease severity in each sheath of inoculated plants were recorded. The total lesion length was the sum of the lesion length of leaves in a culm. The degree of disease severity were assigned as follows (Fig. 2): $0=$ no lesion, $1=$ the appearance of watersoaked lesions, $2=$ the appearance of necrotic lesions, $3=$ less than $50 \%$ necrosis on the leaf cross section, $4=$ more than $50 \%$ necrosis on the leaf cross section, and $5=$ necrosis across the entire leaf section resulting in leaf death. The index of disease susceptibility was calculated as follows: susceptibility index $=\left(5 \mathrm{n}_{5}+4 \mathrm{n}_{4}+\right.$ $\left.3 \mathrm{n}_{3}+2 \mathrm{n}_{2}+1 \mathrm{n}_{1}+0 \mathrm{n}_{0}\right) / 5 \mathrm{~N} \times 100 ;$ where $\mathrm{n}_{0-5}$ is the number of leaves in each degree $(0$ to 5) and $\mathrm{N}$ is the number of total leaves investigated in a culm. All lesions from a culm are added together to give a total lesion length for each culm. Twenty-five replications were used for each cultivar in the cultivar comparison study. The cultivar assay was repeated using both rating methods with similar results. The analysis of variance of the susceptibility index and the lesion length caused by $R$. solani was performed using SAS (version 6.12). Separation of cultivar means for both suscepti- 
bility index and lesion length was performed using Duncan's multiple range test.

\section{RESULTS AND DISCUSSION}

An effective inoculation method is a critical component of an accurate disease assay for quantifying levels of sheath blight resistance among rice cultivars. The type of inoculum used in an inoculation method is a major determinant of infection efficiency. Several previous studies used toothpicks colonized with $R$. solani $(19,20,28)$, sclerotia (25), and infected rice grain-hull mixtures $(9,16,27)$. The time required for these inoculum sources to begin active growth in order to initiate infection can vary widely depending on environmental and pathogen factors. Singh et al. (25) reported that smaller sclerotia infected plants more quickly than larger ones. These authors also reported that infection was most rapid using mycelia and slowest using mature sclerotia, with infection time being intermediate for immature sclerotia (25). These pathogen factors led to additional variation in the infection process and the subsequent disease development that has little to do with host resistance. For this reason, only actively growing sources of $R$. solani inoculum were analyzed in this study.

Mycelial balls produced the longest lesions on rice sheaths 7 days after inoculation; in fact, more than twice that produced by colonized agar blocks or mycelial suspensions (Fig. 3). These results were significant and consistent across all cultivars tested $(P<0.001)$. One possible reason for this is that mycelial balls provide greater fungal biomass than the other two types of inocula. Mycelial suspensions were the least efficient inoculation source, possibly because the mycelium has been broken apart, preventing the allocation of nutrients through the mycelial network to the point of infection. Also, the mycelial suspensions were liquid and their retention at the site of inoculation varied depending on the architecture of the cultivar inoculated. The mycelial suspensions caused greater disease in cultivars where the sheath was tightly attached to the stem than cultivars where the sheath was more loosely attached (data not shown). The colonized agar block contained organized mycelia only on the top surface and did not have the same fungal biomass of the mycelial balls. Also, the nutrients provided by the agar block may have encouraged saprophytic growth in the fungus rather than the development of an infection cushion needed for disease development.

Rapid and uniform infection reduces the variation inherent in the disease assays. In this study, $100 \%$ infection rate was achieved by covering the inoculated sheaths with aluminum foil for 3 days. Pilot studies revealed that aluminum foil was necessary to gain consistent infection and that disease often failed to develop without aluminum foil. The saturating humidity retained by the foil covering was crucial for establishing uniform and consistent infections. Therefore, the foil covering is essential in avoiding the variation in disease development that occurred with previous methods of inoculation. The combination of using actively growing mycelial balls as inoculum and aluminum foil as covering provided the reproducible infection necessary for performing the disease assays. The rapid and uniform infection of host tissues in all inoculations achieved by this assay is critical for quantitative analysis of sheath blight resistance at molecular, biochemical, and genetic levels.
The other methods provided little environmental control during the early infection stage and, thus, are subject to much greater variation than this method using an aluminum foil covering.

Once $R$. solani infection has been established, a precise evaluation method is essential for accurate quantification of disease symptoms. Previous studies have used either lesion length or visual ratings to assess disease severity; however, these methods have not been compared side by side to assess their accuracy. Differences in resistance to sheath blight may be manifested by the rate at which the fungus grows up the sheath and colonizes the

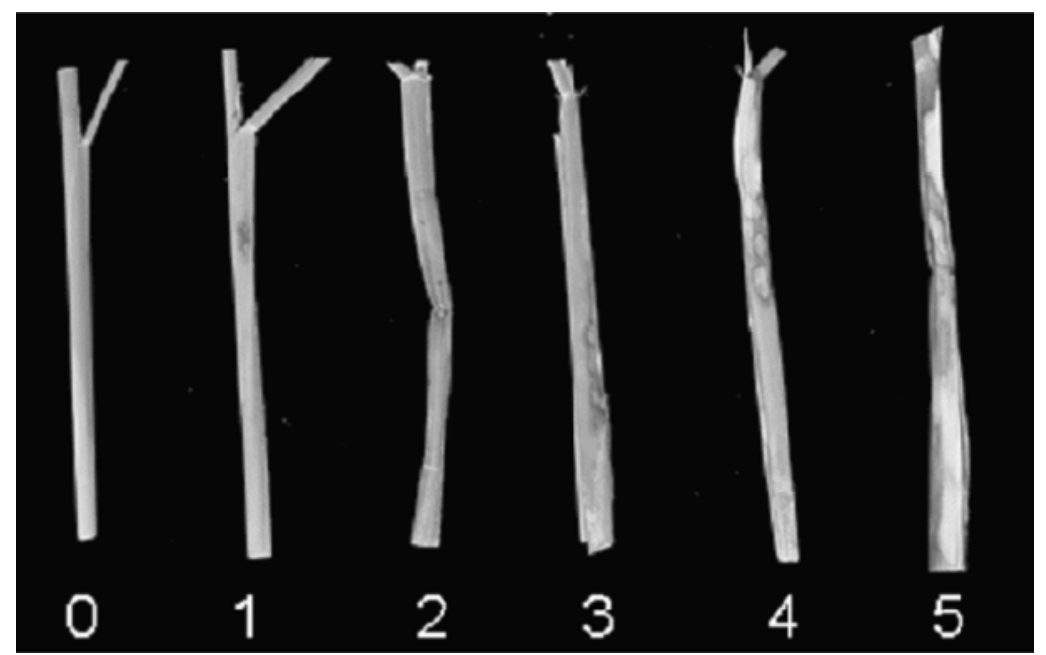

Fig. 2. Representation of sheath blight disease severity rating values. The degree of disease severity were assigned as follows: $0=$ no lesion, $1=$ the appearance of a water-soaked lesion, $2=$ the appearance of necrotic lesion, $3=$ less than $50 \%$ necrosis on the leaf cross section, $4=$ more than $50 \%$ necrosis on the leaf cross section, and $5=$ necrosis across the entire leaf section resulting in leaf death. The susceptibility index of a culm was calculated as follows: susceptibility index $=\left(5 n_{5}+4 n_{4}+3 n_{3}+2 n_{2}\right.$ $\left.+1 n_{1}+0 n_{0}\right) / 5 N \times 100$, where $n_{0-5}$ is the number of leaves in each level ( 0 to 5$)$ and $\mathrm{N}$ is the number of total leaves investigated in a culm.

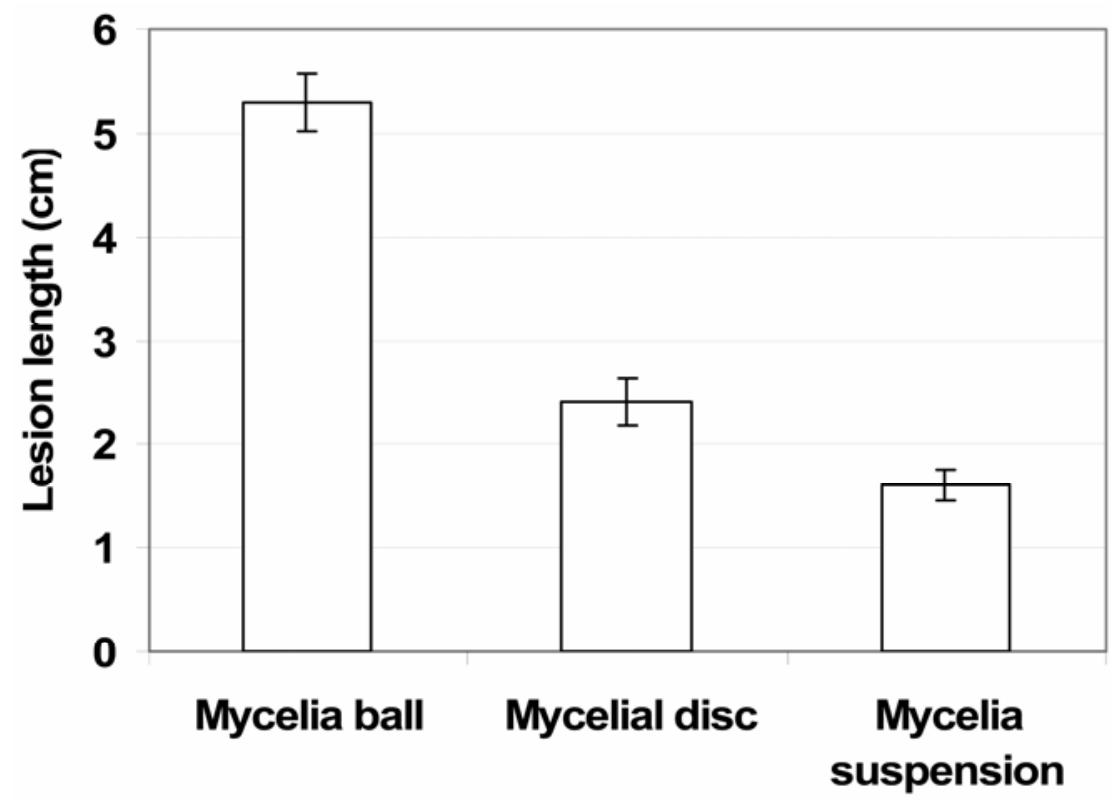

Fig. 3. Comparison of Rhizoctonia solani inoculation methods as measured by mean lesion length 7 days after inoculation across rice cvs. Chucheongbyeo, Junambyeo, Tetep, and Jasmine 85. The bar represents standard error. 
upper leaves of the plant. The rice cultivars used in this study varied widely with regard to sheath blight resistance and agronomic traits such as plant architecture, days to heading, panicle number, and other characteristics (supplemental figure online). The visual ratings of disease were weighted so that the leaves with greatest disease received a propor- tionally higher score than the leaves with less disease.

A comparison of two methods for rating the disease severity was made to determine which method produced the most accurate disease severity data among eight cultivars based on their previously reported susceptibility $(3,9,16,18,28)$. The first method measured total lesion length and the second

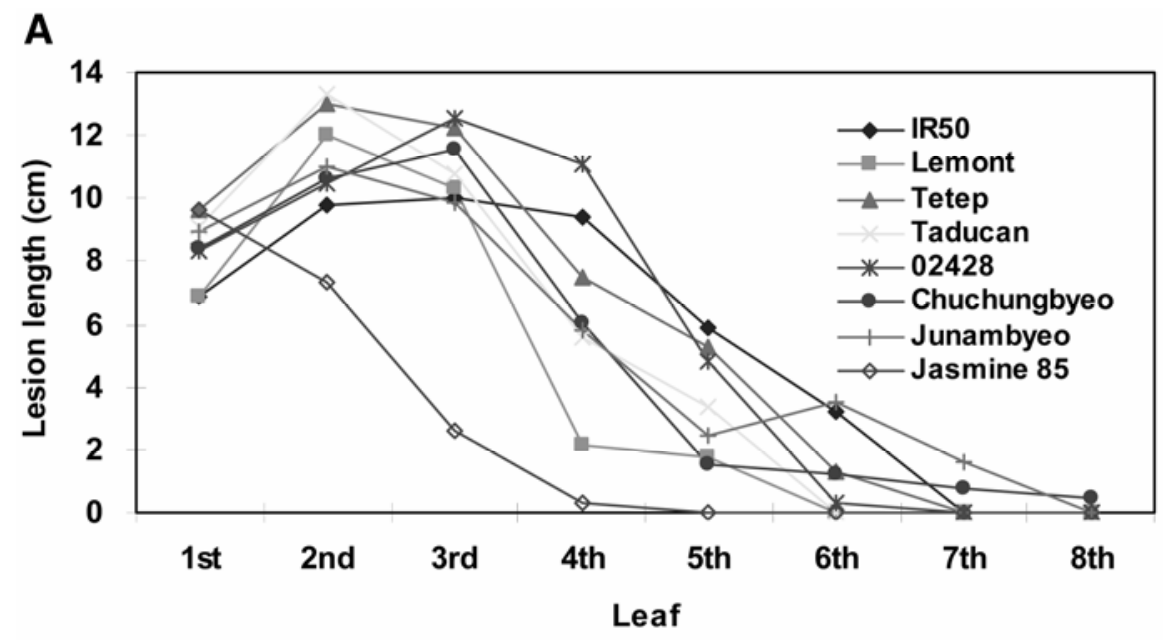

\section{B}

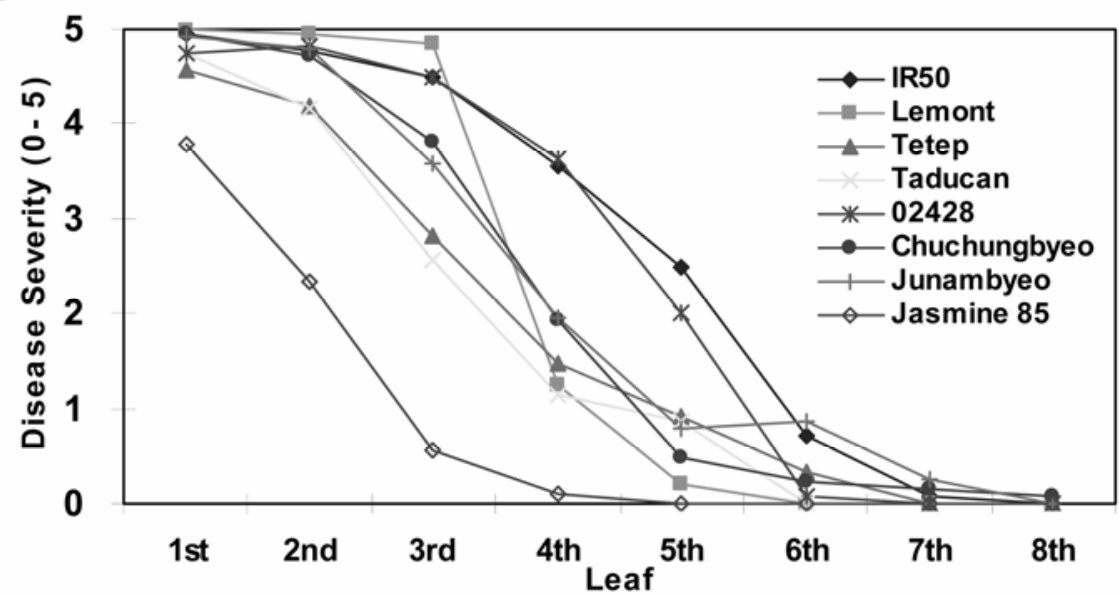

Fig. 4. Cultivar differences of sheath blight severity in each leaf (first leaf is the lowest leaf in a culm) as determined by $\mathbf{A}$, lesion length and $\mathbf{B}$, disease severity index.

Table 1. Cultivar differences in heading date, panicle number, and sheath blight severity as measured by susceptibility index and total lesion length ${ }^{z}$

\begin{tabular}{lccccccc}
\hline \multirow{2}{*}{ Cultivar } & \multicolumn{2}{l}{ Susceptibility index } & & \multicolumn{2}{l}{ Total lesion length } & & \\
\cline { 2 - 3 } & Percent & Rank & & Length $(\mathbf{c m})$ & Rank & Days to heading & Panicles/plant \\
\hline IR 50 & $69.9 \mathrm{a}$ & 1 & & $45.2 \mathrm{abc}$ & 3 & 71 & 15 \\
02428 & $64.9 \mathrm{a}$ & 2 & & $47.6 \mathrm{ab}$ & 2 & 68 & 7 \\
Junambyeo & $56.4 \mathrm{~b}$ & 3 & & $41.6 \mathrm{~cd}$ & 5 & 81 & 12 \\
Lemont & $54.1 \mathrm{~b}$ & 4 & & $33.1 \mathrm{e}$ & 7 & 81 & 9 \\
Chucheongbyeo & $53.7 \mathrm{bc}$ & 5 & & $39.3 \mathrm{~d}$ & 6 & 81 & 14 \\
Tetep & $48.3 \mathrm{~cd}$ & 6 & & $48.9 \mathrm{a}$ & 1 & 87 & 19 \\
Taducan & $44.5 \mathrm{~d}$ & 7 & & $42.3 \mathrm{bcd}$ & 4 & 90 & 25 \\
Jasmine 85 & $17.6 \mathrm{e}$ & 8 & & $19.9 \mathrm{f}$ & 8 & 92 & 14 \\
\hline
\end{tabular}

${ }^{\mathrm{z}}$ Heading date was determined by noting the date when $50 \%$ of the spikelets emerged for a particular cultivar among five plants in individual pots. The panicle number was determined by calculating the mean panicle number among five plants in individual pots. Means having different letters are significantly different (Fisher's least significant difference, $P=0.001$ ) for both lesion length and susceptibility index.

method used the disease susceptibility index. The two disease severity rating systems are shown graphically for both lesion length (Fig. 4A) and disease susceptibility index (Fig. 4B) across each leaf position above the inoculation point for the eight cultivars used in this study. The effect of cultivar was significant for both lesion length and susceptibility index $(P<0.001)$. Although both methods of measuring severity of sheath blight produced the same general trends, the susceptibility index was more clear and consistent across all leaf positions within and across cultivars (Fig. 4A and B). Disease severity decreased as distance from the source of inoculum increased for both evaluation methods (Fig. 4A and B).

When the infected cultivars were ranked, using either total lesion length or disease susceptibility index (Table 1), the susceptibility index rankings were more consistent with the published reports than the lesion length measurement $(3,9,16$, $18,27)$. The susceptibility index also provided a clean differentiation of cultivars based on mean separation (Table 1). Disease severity ratings using lesion length and susceptibility index were highly correlated $\left(R^{2}=0.76\right)$ at each leaf above the point of inoculation, but there were notable differences between the two evaluation methods. The lesion length of the first leaf above the point of inoculation was longer for Jasmine 85 than that of most other cultivars (Fig. 4A). However, the disease susceptibility index in this same cultivar was lower than the other cultivars (Fig. 4B), which is in agreement with the previously published results $(3,9,16,18,28)$. In contrast, the lesion length of the first leaf above the inoculation point for Lemont was shorter than that of the other cultivars (Fig. 4A), but the susceptibility index was higher (Fig. 4B), which also is consistent with the previously published results $(3,9,16,18,28)$.

Overall, the weighted visual rating of disease severity was more accurate in correctly differentiating cultivar difference in sheath blight resistance than the total lesion length on the sheath (Table 1). The difference in plant architecture, namely the length and width of the leaves and the height of the plants among the cultivars, were probably the biggest factors in creating a discrepancy between the two methods. For example, the leaves of IR50 are shorter than those of most other cultivars, and so are the length of the lesions produced by $R$. solani; however, IR50 is one of the most susceptible rice cultivars in existence. IR50 is also one of the shortest cultivars used in this study. On the other hand, the leaf sheath of Tetep is relatively long compared with the other cultivars, and the lesion caused by sheath blight is also longer on this cultivar than other cultivars. However, the susceptibility index as measured by leaf damage and death was lower than for most of the other cultivars tested in the study. 
The shape of the lesion and the width of the leaf also are important factors in measuring disease severity caused by sheath blight. A long narrow lesion will be much less damaging to the flow of solutes up and down the leaf than a short and wide lesion, but will be considered to be more severe by the measurement of lesion length. The measurement of lesion width also brings up the problem of plant architecture differences among the cultivars. A cultivar with a wide leaf sheath may have a much wider lesion than a cultivar with a narrow leaf sheath but still retain a sufficient flow of nutrients through the leaf. Lesion length does not determine the likelihood of leaf death, but the visual ratings of lesion severity does. Plant architecture also can vary among plants of the same cultivar due to environmental effects, emphasizing the need for an evaluation method that is independent of this source of variation. As a result, the disease susceptibility index is a more accurate measure of disease severity and is not skewed by cultivar differences in plant architecture as are direct measurements of lesion length.

Several agronomic traits such as heading date, plant height, and panicle number have been reported to be linked to sheath blight resistance or susceptibility $(11,12$, $18,28)$. In addition to disease severity, the number of panicles and heading date were cataloged for all the cultivars used in this study. Fewer days to heading and susceptibility to sheath blight were positively correlated $\left(R^{2}=0.74, R^{2}=0.91\right.$ without Jasmine 85). Cultivars heading sooner generally were more susceptible to sheath blight than those heading later (Table 1). Resistance to sheath blight and heading date are the phenotypes that may be genetically linked (18). The relationship between panicle number and sheath blight susceptibility was not apparent in this study. Overall, cultivars that had a late heading date generally were more resistant to sheath blight (Table 1). These results are similar to previously published reports $(11,12,18,28)$.

In summary, the selection of resistant cultivars is the most economically and environmentally beneficial way of reducing losses to sheath blight in rice. Cultural control methods $(19,21)$ are insufficient and the use of fungicides (2) may not be economically or environmentally sustainable. Transformation of rice cultivars with defense genes has provided only partial resistance (7). A concerted effort is currently underway in the United States to identify QTL for sheath blight resistance and to study the key genes and underlying mechanisms of the disease resistance. To achieve these goals, uniform and effective infection and accurate evaluation methods are required for detailed genetic, molecular, biochemical, and functional genomic analyses and for measuring quantitative differences in sheath blight resistance among rice breeding lines, mutants, and transgenic plants. Most recently, a quick method has been developed to screen rice cultivars for sheath blight resistance during the seedling stage (6). In this study, improved methods were developed for highly consistent and reproducible inoculation of sheath blight pathogen and for more accurate measurement of disease severity regardless of plant architecture. Because the disease assay uses rice plants at late tillering stage, it requires a longer duration of time. However, the disease assay may complement the seedling-based quick screening (6) and allow further verification of sheath blight resistance in rice plants.

\section{ACKNOWLEDGMENTS}

This study was supported by Korea Research Foundation oversea training fellowship (M01-2005000-10120-0 to D.-S. Park) and the United States Department of Agriculture (USDA)/NRI Rice Coordinated Agricultural Project grant (200435317-14867 to Y. Yang). We thank Y. Jia at USDA-Agricultural Research Service Dale Bumpers National Rice Research Center for providing $R$. solani isolate RR0140 and R. McNew at the University of Arkansas for assistance with the statistical analysis.

\section{LITERATURE CITED}

1. Eizenga, G. C., Lee, F. N., and Rutger, J. N. 2002. Screening Oryza species plants for rice sheath blight resistance. Plant Dis. 86:808-812.

2. Groth, D. E. 2005. Azoxystrobin rate and timing effects on rice sheath blight incidence and severity and rice grain and milling yields. Plant Dis. 89:1171-1174.

3. Groth, D. E., and Norwick, E. M. 1992. Selection for resistance to rice sheath blight through number of infection cushions and lesion type. Plant Dis. 76:721-723.

4. Groth, D. E., Rush, M. C., and Lindberg, G. D. 1990. Foliar fungicides for control of rice diseases in the United States. Pages 31-52 in: Pest Management in Rice. B. T. Grayson, ed. Elsevier Applied Science, London and New York.

5. Hashiba, T. 1984. Estimating method of severity and yield loss by rice sheath blight disease. Bull. Hokuriku Nat. Agric. Stn. 26:115-164.

6. Jia, Y., Correa-Victoria, F., McClung, A., Zhu, L., Liu, G., Wamishe, Y., Xie, J., Marchetti, M.A., Pinson, S. R. M., Rutger, J. N., and Correll, J. C. 2007. Rapid determination of rice cultivar responses to the sheath blight pathogen Rhizoctonia solani using a micro-chamber screening method. Plant Dis. 91:485-489.

7. Kalpana, K., Maruthasalam, S., Rajesh, T., Poovannan, K., Kumar, K.K., Kokiladevi, E., Raja, J.A.J., Sudhakar, D., Velazhahan, R., Samiyappan, R., and Balasubramanian, $\mathrm{P}$. 2006. Engineering sheath blight resistance in elite indica rice cultivars using genes encoding defense proteins. Plant Sci. 170:203-215.

8. Khush, G. S. 1977. Disease and insect resistance in rice. Adv. Agron. 29:268-341.

9. Kim, K. H., and Rush, M. C. 1986. Inheritance of infection cushion formation by Rhizoctonia solani Kühn on rice leaf sheath. Korean J. Breed. 18:167-173.

10. Lee, F. N., and Rush, M. C. 1983. Rice sheath blight: a major rice disease. Plant Dis. 67:829832 .

11. Li, Z., Pinson, S. R. M., Marchetti, M. A., Stansel, J. W., and Park, W. D. 1995. Characterization of quantitative trait loci (QTLs) contributing to field resistance to sheath blight (Rhizoctonia solani). Theor. Appl. Genet.
91:382-388.

12. Li, Z., Pinson, S. R. M., Stansel, J. W., and Park, W. D. 1995. Identification of quantitative trait loci (QTLs) for heading date and plant height in cultivated rice (Oryza sativa L.). Theor. Appl. Genet. 91:374-381.

13. Marchetti, M. A., and Bollich, C. N. 1991. Quantification of the relationship between sheath blight severity and yield loss in rice. Plant Dis. 75:773-774.

14. Masajo, T. M. 1976. Varietal Resistance in Rice to Sheath Blight by Thanatephorus $\mathrm{Cu}$ cumeris (Frank) Donk (Rhizoctonia solani Kühn). Louisiana State University, Baton Rouge.

15. Ou, S. H. 1985. Rice Diseases, 2nd ed. Commonwealth Mycological Institute, Kew, England.

16. Pan, X. B., Rush, M. C., Sha, X. Y., Xie, Q. J., Stetina, S. D., and Oard, J. H. 1999. Major gene, nonallelic sheath blight resistance from the rice cultivars 'Jamine 85 ' and 'Tequing'. Crop Sci. 39:338-346.

17. Parmeter, J. R. 1970. Biology and Pathology of Rhizoctonia solani. University of California Press, Berkeley.

18. Pinson, S. R. M., Capdevielle, F. M., and Oard, J. H. 2005. Confirming QTLs and finding additional loci conditioning sheath blight resistance in rice using recombinant inbred lines. Crop Sci. 45:503-510.

19. Rodrigues, F. A., Datnoff, L. E., Korndorfer, G. H., Seebold, K. W., and Rush, M. C. 2001. Effect of silicon and host resistance on sheath blight in rice. Plant Dis. 85:827-832.

20. Rodrigues, F. Á., Vale, F. X. R., Datnoff, L. E. Prabhu, A. S., and Korndörfer, G. H. 2003. Effect of rice growth stages and silicon on sheath blight development. Phytopathology 93:256261.

21. Rush, M. C., and Lee, F. N. 1992. Sheath blight. Pages 22-23 in Compendium of Rice Diseases. R. K. Webster and P. S. Gunnell, eds. The American Phytopathology Society, St. Paul, MN.

22. Sato, H., Ideta, O., Ando, I., Kunihiro, Y., Hirabayashi, H., Iwano, M., Miyasaka, A. Nemoto, H., and Imbe, T. 2004. Mapping QTLs for sheath blight resistance in the rice line WSS2. Breed. Sci. 54:265-271.

23. Savary, S., and Mew, T. W. 1996. Analyzing crop losses due to Rhizoctonia solani: Rice sheath blight, a case study. Pages 237-244 in Rhizoctonia Species: Taxonomy, Molecular Biology, Ecology, Pathology and Disease Control. B. Sneh, S. Jabaji-Hare, S. Neate, and G. Dijst, ed. Kluwer Academic Publisher, Dordrecht, The Netherlands.

24. Sha, X. Y., and Zhu, L. H. 1989. Resistance of some rice varieties to sheath blight $(\mathrm{ShB})$. Int. Rice Res. Newsl. 15:7-8.

25. Singh, A., Rohilla, R., Singh, U. S., Savary, S., Willocquet, L., and Duveiller, E. 2001. An improved inoculation technique for sheath blight of rice caused by Rhizoctonia solani. Can. J. Plant Pathol. 24:65-68.

26. Wasano, K., Oro, S., and Kido, Y. 1983. The syringe inoculation method for selecting rice plants resistant to sheath blight, Rhizoctonia solani Kühn. Japan. J. Trop. Agric. 27:131139.

27. Willocquet, L., Fernandez, L., and Savary, S. 2000. Effect of various crop establishment methods practiced by Asian farmers on epidemics of rice sheath blight caused by Rhizoctonia solani. Plant Pathol. 49:346-354

28. Zou, J. H., Pan, X. B., Chen, Z. X., Xu, J. Y., Lu, J. F., Zhai, W. X., and Zhu, L. H. 2000 Mapping quantitative trait loci controlling sheath blight resistance in two rice cultivars (Oryza sativa L.). Theor. Appl. Genet. 101:569-573. 